\title{
The acceptance of radiographers to use Non-fluoroscopy Guided Positioning and the influence of leadership: a survey research
}

\author{
S. Germonpré ${ }^{1, *}$, G. Vermaut ${ }^{2}$ and J. Trybou ${ }^{2}$ \\ ${ }^{1}$ Medical Imaging, Department of Healthcare, Odisee University-College, Blekerijstraat, 23-29, 1000, Brussels, Belgium. \\ 2 Department of Public Health, Ghent University, De Pintelaan, 185, 9000, Ghent, Belgium.
}

Received: 11 May 2018 / Accepted: 3 July 2019

\begin{abstract}
Although using fluoroscopy to position the patient for radiographic examinations cannot be justified, it is commonly used by several radiology departments. In an attempt to understand the determinants of non-fluoroscopy-guided positioning, this study investigates the predictive value of performance expectancy, effort expectancy and social influence. Subsequently, the moderation of individualised consideration by the head radiographer, as a leadership trait, is tested. The study is performed by using data of a self-administered paper and pencil questionnaire distributed among 17 Belgian hospitals. All 301 radiographers working in radiology departments were invited to cooperate. We found that effort expectancy is the strongest predictor, closely followed by performance expectancy. The effect of social influence is also found to be significant. The outcome of the moderation analysis shows that only the effect of social influence on the behavioural intention to use non-fluoroscopy-guided positioning is significantly moderated by individualised consideration. In conclusion we can state that the findings of this research can be a helpful tool for radiologists and head radiographers to stimulate the staff to improve imaging practices.
\end{abstract}

Keywords: radiography / fluoroscopy / patient protection / quality assurance

\section{Introduction}

Exposure to medical radiation is considered one of the most serious patient safety issues (IAEA, 2015). Accordingly, many countries have identified the reduction of radiation exposure as a priority for quality improvement (i.e. Vrijens et al., 2012). Minimizing the patient dose, following the ALARA-principle (the radiation dose should be As Low As Reasonably Achievable), is extremely important to minimize the risk of adverse stochastic effects, such as cancer (Statkiewicz-Sherer et al., 2014).

From this perspective, the question whether or not to use fluoroscopy for the patient's positioning is subject of discussion. Fluoroscopy-guided positioning is the practice of using fluoroscopy to determine the correct position and location of the anatomy of the patient before making the diagnostic image. This practice can lead to a greatly increased patient dose of radiation (Dierckx et al., 2005) and therefore should not be used as a substitute for adequate positioning skills. Although FGP is a violation of the ALARA radiation safety principles and is seen by the American Society of Radiologic Technologists (ASRT) as unethical (ASRT, 2017),

*Corresponding author: sofie.germonpre@odisee.be it is commonly used (Deprez et al., 2001; Saunders et al., 2005; Ciraj-Bjelac et al., 2011; Germonpré et al., 2016).

The non-use of FGP (henceforth "non-FGP") on the other hand, relies heavier on the radiographer's skills to position the patient precisely. Non-FGP is based on the basic principle of positioning the patient carefully by the use of anatomic landmarks in order to limit the radiation exposure to the patient (Bontrager and Lampignano, 2014).

According to the literature, FGP as positioning aid technique can be justified for specific radiographic examinations with a relatively high level of complexity (i.e. the lateral L5-S1 projection) (Saunders et al., 2005). The technique is used for an ultrashort fluoroscopic pulse (Deprez et al., 2001), in order to reduce the amount of retakes and consequently minimize patient radiation dose. However, in most Belgian radiology departments radiographers routinely use FGP for almost all radiographies (with the exception of relatively easy small bone examinations such as the hand or wrist). Not surprisingly, a study in Belgium found that the use of fluoroscopy for positioning the patient can greatly increase patient dose of radiation (Dierckx et al., 2005). The argument in favour of FGP becomes tenuous if held against current data: with repeat rates of barely 7 to $8 \%, 90 \%$ of all patients would be overexposed unnecessarily when using fluoroscopy (Statkiewicz-Sherer et al., 2014). 
Although the risk of harmful effects of low dose exposures is relatively low, the ALARA principle (Statkiewicz-Sherer et al., 2014) posits that the patient dose should always be kept to a minimum. Moreover, it is possible that throughout time patients are imaged multiple times and thus are exposed multiple times and cumulate these radiation doses. Therefore, since it is possible to perform radiographies without the additional radiation dose exposure induced by the use of fluoroscopy, it can be argued that the routine use of FGP for radiographies cannot be justified (ASRT, 2017). Non-FGP is an important strategy in minimizing the patient dose in radiography. Therefore measures must be taken to increase the uptake of non-FGP practice.

The objective of this paper is to examine the determinants of the use of non-fluoroscopy-guided positioning (FGP) in Belgian radiology departments. More specifically, this study aims to investigate the effect of performance expectancy (PE), effort expectancy (EE), social influence (SI) and leadership on the behavioural intention to use non-FGP. To study the effect of PE, EE and SI we used the theoretical model of the Unified Theory of Acceptance and Use of Technology (UTAUT) (Venkatesh et al., 2003). To study the impact of leadership on the intention to use non-FGP, we used the concept "individualized consideration" (Avolio and Bass, 2004). UTAUT was initially designed to predict the acceptance and use of a technology (e.g. electronic patient records). To our knowledge, this study is the first study that applied the UTAUT framework to examine the acceptance and use of adequate professional practices (i.e. non-FGP practices). In addition previous research found that leadership is important in improving the quality of care and patient safety (Kroch et al., 2006; Jiang et al., 2008; Germonpré et al., 2016). From these insights we also studied if leadership has an influence in the context of radiography, more precisely on the radiographers' use of nonFGP. Finally this is the first study investigating the determinants of the use of non-FGP in a quantitative way (Germonpré et al., 2016). This study should increase radiologists' and radiographers' knowledge of the determinants of non-FGP positioning and enable them to improve current practices.

\section{Theoretical framework}

\subsection{The Unified Theory of Acceptance and Use of Technology (UTAUT)}

The theoretical model used in this article is the Unified Theory of Acceptance and Use of Technology (UTAUT) (Venkatesh et al., 2003).

The UTAUT-model consists of four main predictors of the behavioural intention to use a technology and ultimately actual use. The determinants are performance expectancy (PE), effort expectancy (EE), social influence (SI) and facilitating conditions (FC). This study (see Fig. 1) focuses on the effect of PE, EE and SI. Facilitating conditions were not included, because this was not a discriminating factor (all departments had adequate X-ray machines as well as positioning aids).

The first determinant, performance expectancy can be defined as "the degree to which the user expects that using the system will help him or her to attain gains in job performance" (Venkatesh et al., 2003, p. 447). Research shows that this is one

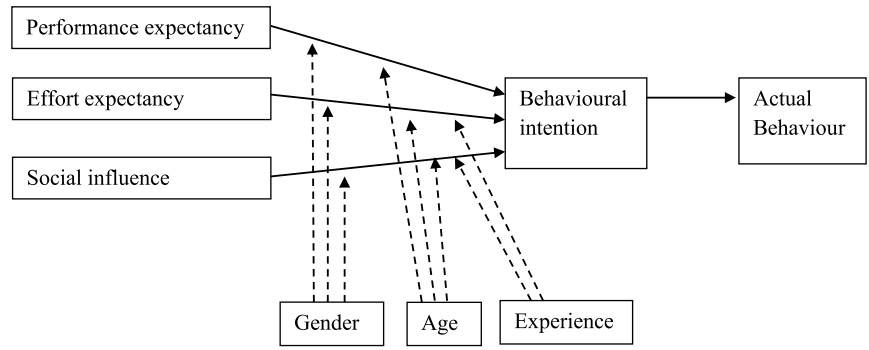

Fig. 1. Schematic view of the research model, based on the original UTAUT. Full lines are the effects tested in this study.

of the most important predictors of the intention to use technology (Venkatesh et al., 2003; Chang et al., 2007; Kijsanayotin et al., 2009; Liu et al., 2014). Subsequently effort expectancy can be explained as "the degree of ease associated with the use of the system" (Venkatesh et al., 2003, p. 450). Social influence refers to "the degree to which an individual perceives that important others believe he or she should use the new system" (Venkatesh et al., 2003, p. 451).

The outcome variables in the UTAUT are behavioural intention and actual behaviour. According to previous research there is a strong and significant relationship between behavioural intention and the behaviour as such (Sheppard et al., 1988; Venkatesh et al., 2003). Also in healthcare settings this relationship is conclusive (Chau and $\mathrm{Hu}, 2001$ ). More specifically the study of Pynoo et al. (2012) about physicians' acceptance and use of Picture Archiving and Communication System (PACS) found a significant relationship between the acceptance and use of PACS shortly after the introduction period).

\subsection{Leadership}

Studies have proven the importance of leadership in improving the quality of care and patient safety (Kroch et al., 2006; Jiang et al., 2008). More precisely, in previous qualitative research (Germonpré et al., 2016) clinical leadership of radiologists and the head radiographer was identified as an important determinant for using non-FGP. This includes appreciative coaching and guidance on the part of the radiologists and the head radiographer.

Therefore, in this research, we focus on the possible impact of individualised consideration of the head radiographer on the use of non-FGP positioning techniques. Individualised consideration can be understood as the characteristic of leaders who pay attention to the individual needs of the team members and coach them through obstacles and difficult situations (Avolio and Bass, 2004; Gabel, 2012). Those leaders reinforce the' strengths and self-efficacy of team members within a supportive climate (Avolio and Bass, 1995).

Based on previous literature this study hypothesizes a moderation of individualised consideration on the effects of the three predictors on behavioural intention (Fig. 2).

\section{Material and methods}

The aim of this study is to gain knowledge into the acceptance of non-FGP in Belgian radiology departments. 


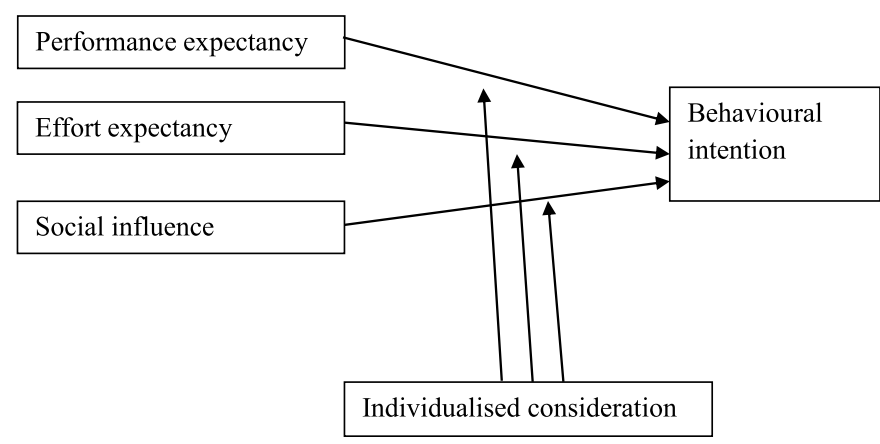

Fig. 2. Schematic view of the moderation effects tested in this study.

The effect of performance expectancy, effort expectancy and social influence on the behavioural intention to use non-FGP will be further explored. Furthermore, the possible influence of individualised consideration on these effects will be examined.

Based on previous research the following hypotheses (Hs) were formulated:

- H1-3: PE (H1), EE (H2), SI (H3) is expected to have a positive effect on the behavioural intention to use nonFGP;

- H4: The behavioural intention to use non-FGP is expected to have a positive effect on the actual use of the technique;

- H5-H7: The effect of PE (H5), EE (H6) and SI (H7) on the behavioural intention to use non-FGP is expected to be moderated by individualised consideration.

\subsection{Sample and procedure}

Out of 17 randomly addressed hospitals in the Flemish part of Belgium, 15 agreed to cooperate. Paper and pencil questionnaires were distributed among 301 radiographers in 2015. This study focuses on the following six concepts: performance expectancy, effort expectancy, social influence, behavioural intention, actual use and individualised consideration. Approval by the Ethics Committee was obtained. Participation was voluntary and all information that could identify the subjects was removed.

\subsection{Questionnaire}

The questionnaire consisted of 26 items and was divided into two sections. The first section contained questions about demographic variables. Date of birth, diploma, sex, years of employment, time working at the radiology department, time working in radiography were asked. In addition they had to answer if they had followed an external training in non-FGP.

The second section contained questions about the research variables. The items of PE, EE, SI and behavioural intention were formulated based on the original questionnaire by Venkatesh et al. (2003). For those four concepts, participants scored their level of agreement using a seven-point Likert scale, ranging from 1 totally disagree to 7 totally agree. One item concerning the actual use of non-FGP was assessed on a
Table 1. Results of the reliability analysis (Cronbach's Alpha).

\begin{tabular}{lll}
\hline Construct & $\begin{array}{l}\text { Cronbach's } \\
\text { Alpha }\end{array}$ & $\begin{array}{l}\text { Number } \\
\text { of items }\end{array}$ \\
\hline Performance expectancy & 0.84 & 3 \\
Effort expectancy & 0.86 & 4 \\
Social influence & 0.80 & 4 \\
Behavioural intention & 0.94 & 3 \\
Individualised consideration & 0.80 & 4 \\
\hline
\end{tabular}

seven-point Likert scale, ranging from 1 never to 7 always. The leadership concept in this study was approached from a particular leadership trait, being individualised consideration (Avolio and Bass, 2004). Subjects scored their level of agreement on four items using a five-point Likert scale, ranging from 1 totally absent to 5 almost always present.

All question items in this study were translated into Dutch by two independent translators and then, as a control, translated back blindly by a third translator. Minor adaptions were made to fit in our study. All items of the second section are presented in Appendix A.

\section{Results}

\subsection{Reliability analysis}

The survey was collated from previously published instruments that have demonstrated sound psychometric properties in past research. The Cronbach's Alphas showed satisfactory levels of reliability (above a 0.7 threshold) (Nunnally, 1978) of the constructs (Tab. 1).

\subsection{Descriptive research}

\subsubsection{Demographic variables}

A total of 177 subjects (114 women and 63 men) completed the questionnaire, a response rate of $58.8 \%$. The average age of the participants was 37.81 years $(\mathrm{SD}=11.27)$. Fifty-nine employees were nursing assistants, 112 had a bachelor degree ( 75 nurses and 37 radiographers) and six did not fill in the question. Sixty-one subjects completed an external training in non-FGP.

\subsubsection{Research variables}

The majority of the participants answered that they regularly $(28.2 \%)$ or frequently $(27.7 \%)$ used non-FGP to do radiographic examinations (Tab. 2).

In order to comprehensibly summarize the scores of the three predictors and behavioural intention, following groups are introduced: Group 1: all participants with a general score between 1 and 3.49; Group 2 all scores rounded up to four; Group 3: scores between 4.5 and 7. For the concept of individualised consideration again three groups are introduced: Group 1: all participants with a general score between one and 2.49; Group 2: all scores rounded up to three and Group 3: all scores between 3.5 and 5. All frequencies and percentages of the concepts are summarized in Table 2. 
Table 2. Summary of all research variables.

\begin{tabular}{|c|c|c|}
\hline & Scale options & $\begin{array}{l}\text { Frequencies } \\
\text { (percentages) }\end{array}$ \\
\hline \multirow{7}{*}{ Use of non-FGP } & Never & $4(2.3)$ \\
\hline & Sporadically & $9(5.1)$ \\
\hline & Sometimes & $18(10.2)$ \\
\hline & Regularly & $50(28.2)$ \\
\hline & Frequently & $49(27.7)$ \\
\hline & Mostly & $42(23.7)$ \\
\hline & Always & $5(2.8)$ \\
\hline \multirow{4}{*}{$\begin{array}{l}\text { Performance } \\
\text { expectancy }\end{array}$} & G1: Totally disagree to disagree & $29(16.4)$ \\
\hline & G2: Neutral & $39(22.0)$ \\
\hline & G3: Agree to totally agree & $104(58.8)$ \\
\hline & Missing & $5(2.8)$ \\
\hline \multirow{4}{*}{ Effort expectancy } & G1: Totally disagree to disagree & $21(11.9)$ \\
\hline & G2: Neutral & $55(31.1)$ \\
\hline & G3: Agree to totally agree & $88(49.7)$ \\
\hline & Missing & $13(7.3)$ \\
\hline \multirow{4}{*}{ Social influence } & G1: Totally disagree to disagree & $61(34.5)$ \\
\hline & G2: Neutral & $73(41.2)$ \\
\hline & G3: Agree to totally agree & $33(18.6)$ \\
\hline & Missing & $10(5.6)$ \\
\hline \multirow{4}{*}{$\begin{array}{l}\text { Behavioural } \\
\text { intention }\end{array}$} & G1: Totally disagree to disagree & $87(49.2)$ \\
\hline & G2: Neutral & $52(29.4)$ \\
\hline & G3: Agree to totally agree & $31(17.5)$ \\
\hline & Missing & $7(4.0)$ \\
\hline \multirow{4}{*}{$\begin{array}{l}\text { Individualised } \\
\text { consideration }\end{array}$} & G1: Totally absent to rarely & $30(16.9)$ \\
\hline & G2: Sometimes & $57(32.2)$ \\
\hline & G3: Frequently to always & $77(43.5)$ \\
\hline & Missing & $13(7.3)$ \\
\hline
\end{tabular}

\subsection{Regression analysis}

In order to test $\mathrm{H} 1, \mathrm{H} 2$ and $\mathrm{H} 3$, a linear regression was conducted. Based on previous literature (Venkatesh et al., 2003) the variables sex and years of employment are proven to moderate the effect of the independent variables on behavioural intention. Therefore, their effect is neutralized by entering them in the regression. Table 3 presents the results of the regression analysis with behavioural intention as dependent variable. Performance expectancy $(t=-4.10$, $p<0.01)$ as well as effort expectancy $(t=4.65, p<0.01)$ have a significant effect on behavioural intention to use nonFGP for all radiographs. The influence of social influence on behavioural intention is marginally significant $(t=1.95$, $p<0.10$ ).

To test the effect of behavioural intention on actual use (H4), a linear regression was conducted. Based on research of Venkatesh et al. (2003) there is no need to control for sex and years of employment. The effect of behavioral intention on actual use is statistically significant $(b=0.31, t=4.64$, $p<0.01)$.

\subsection{Moderation analysis}

In order to investigate if there is an influence of leadership on the effect of the three predictors on behavioural intention
Table 3. Results of the regression analysis with behavioural intention (BI) as dependent variable.

\begin{tabular}{lccrrl}
\hline Model & B & SE B & B & \multicolumn{1}{c}{ T } & \multicolumn{1}{c}{ P } \\
\hline (Constant) & 1.86 & 0.75 & & 2.50 & $0.01^{* *}$ \\
Sex & 0.24 & 0.20 & 0.08 & 1.16 & 0.25 \\
Years of employment & 0.03 & 0.08 & 0.03 & 0.37 & 0.71 \\
PE & -0.29 & 0.07 & -0.29 & -4.10 & $0.00^{* *}$ \\
EE & 0.45 & 0.10 & 0.35 & 4.65 & $0.00^{* *}$ \\
SI & 0.17 & 0.09 & 0.14 & 1.94 & $0.05^{*}$ \\
\hline
\end{tabular}

$*=p<0.10 ; * *=p<0.01$.

(H5, H6 and H7), a moderation analysis was performed. In a first step the three independent variables and individualised consideration were centralized. In a second step, the interaction terms were created. The third and last step included the regression analysis. Results of the moderation analysis are displayed in Table 4 . The four main variables had a significant effect. Performance expectancy $(t=-3.41$, $p<0.01)$ and individual consideration $(t=-1.90, p<0.10)$ had a significant negative effect whereas effort expectancy $(t=3.70, p<0.01)$ and social influence $(t=3.02, p<0.01)$ had a significant positive effect. The interaction effects of performance expectancy and individual consideration $(t=0.93, p>0.10)$, and effort expectancy and individual consideration $(t=-1.13, p>0.10)$ did not achieve significance. The interaction effect of social influence and individual consideration was marginally significant $(t=1.83, p<0.10)$.

\section{Discussion}

The paper's first aim is to investigate the three predictors (PE, EE and SI) of the acceptance of non-FGP for radiographs in Belgian radiology departments. As a second aim, this study wants to explore the influence of leadership on the effect of those three concepts on the behavioural intention to use nonFGP.

The respondents' years of employment in radiography equals years of experience, which is seen as a moderator of the effect of EE and SI on behavioural intention (Venkatesh et al., 2003). In this sample, almost three fourth of the participants had five or more years of relevant experience. Although $66 \%$ of the participants worked fulltime at the radiology department, only $34 \%$ of all participants worked fulltime in radiography. Since acquiring positioning skills is found to be the result of long and intensive practice (Germonpré et al., 2016), all those employees who worked only part-time in radiography cannot totally be blamed for not using such a difficult technique. In addition only a minority of $35 \%$ completed an external programme. Previous research suggested that higher educated subjects might differ from others, in that they comprehend a complex technique more quickly and are less influenced by subjective norms (Chau and $\mathrm{Hu}, 2001$; Chang et al., 2007). Moreover, Dierckx et al. (2005) concluded that, in Belgium, the decision to position 
Table 4. Results of the moderation analysis.

\begin{tabular}{lrlrrl}
\hline Model & \multicolumn{1}{c}{ B } & SE B & \multicolumn{1}{l}{ B } & \multicolumn{1}{l}{ T } & P \\
\hline (Constant) & 3.31 & 0.32 & & 10.20 & $0.00^{* *}$ \\
Sex & 0.16 & 0.21 & 0.06 & 0.75 & 0.45 \\
Years of & -0.01 & 0.08 & -0.01 & -0.07 & 0.95 \\
employment & & & & & \\
PE_C & -0.25 & 0.07 & -0.25 & -3.41 & $0.00^{* *}$ \\
EE_C & 0.39 & 0.10 & 0.29 & 3.70 & $0.00^{* *}$ \\
SI_C & 0.29 & 0.10 & 0.24 & 3.02 & $0.00^{* *}$ \\
IC_C & -0.21 & 0.11 & -0.14 & -1.90 & $0.06^{*}$ \\
PE_C*IC_C & 0.07 & 0.07 & 0.07 & 0.93 & 0.36 \\
EE_C*IC_C & -0.11 & 0.10 & -0.09 & -1.13 & 0.26 \\
SI_C*IC_C & 0.16 & 0.09 & 0.14 & 1.83 & $0.07 *$ \\
\hline
\end{tabular}

$*=p<0.10 ; * *=p<0.01$.

the patient with or without fluoroscopy is determined by the staff's training.

Use of non-FGP as an outcome variable, was measured by one item. Although the adverse effect of radiation is proven and FGP is seen as unethical (Berrington de González and Darby, 2004; IAEA, 2015), still half of the sample failed to use non-FGP.

The second outcome variable is the behavioural intention to position a patient without fluoroscopy. Almost 30\% was not sure about what they would do and nearly half admitted that they did not intend to use non-FGP to position the patient. Earlier we found that only $35 \%$ completed an external training. Insufficient knowledge and skills could be one possible explanation for not using anatomic landmarks to position the patient. We argue that sufficient knowledge and skills regarding non-FGP are needed for the acceptance and use of non-FGP. We believe that a theoretical course in non-FGP in combination with practical internal training and continuous coaching are important measures to increase the acceptance and use of non-FGP. In order to assure a rapid effective learning process, we argue that, during the learning period, radiographers must work continuously in radiography. In addition we believe that working fulltime or most of the working hours in radiography is important to keep radiographers skilled in non-FGP.

Another likely explanation for not using non-FGP can be found in the non-monitoring of the radiologist and/or head radiographer, especially in institutions where fluoroscopy is allowed to position the patient. According to the ASRT only the use of non-FGP for radiographies can be justified (ASRT, 2017), so the use of fluoroscopy should not be allowed. However in some literature it is argued that the use of fluoroscopy could prevent retakes and as a consequence could minimize patient radiation dose (Deprez et al., 2001; Saunders et al., 2005; Haynes and Curtis, 2009). This was also one of the findings of our previous qualitative study (Germonpré et al., 2016) in which radiographers mentioned that fluoroscopy can be useful for patients with anatomical abnormalities and for complex imaging procedures (e.g. oblique or lateral image of the spine). However, independent of the fact that radiology departments allow or don't allow FGP, a good monitoring system of the fluoroscopy time and retake rate is necessary, in order to detect and optimize difficulties in radiographic positioning. In addition we believe that without a good monitoring system in radiology departments where FGP is allowed for certain exposures, positioning skills will fade or will not develop. In order to acquire adequate positioning skills the staff should be supervised and fluoroscopy time should be measured (Germonpré et al., 2016).

The first predictor of the behavioural intention to position the patient without fluoroscopy is PE. Contrary to the other concepts, the items that represent PE are formulated in favour of FGP. The majority of the respondents agreed on the fact that the use of fluoroscopy entails a rise in job performance. This is a remarkable fact given the negative recommendations in the literature (Berrington de González and Darby, 2004; IAEA, 2015). It is possible that the participants answered these items without considering the negative consequences of FGP. Another possible reason for considering FGP as better job performance, could be that the radiographers have the opinion that by using fluoroscopy work goes faster (Germonpré et al., 2016). In addition to this it is important to mention that X-ray devices with fluoroscopy ability are not practical to work with when performing non-FGP. From this we can assume that the majority of the respondents works on radiology departments where the radiographies must be performed on X-ray devices with fluoroscopy ability. In order to change radiographers' attitude with respect to non-FGP the implementation of more $\mathrm{X}$-ray devices without fluoroscopy ability and the obligation to perform the radiographies on these devices are important measures.

Concerning EE, the participants seemed to be considering the technique of non-FGP as rather simple. These results are in contrast with the scores on the item about the actual use of nonFGP. Although only a few people seemed to think that the technique was hard, still half of the participants did not position the patients without the use of fluoroscopy. In most Belgian radiology departments radiographers routinely use non-FGP for almost all small bone examinations of the upper and lower extremities (Germonpré et al., 2016). It is possible that the respondents answered the $\mathrm{EE}$ items for the examinations that they routinely perform without fluoroscopy. The contrast with the scores for actual use and intention to use non-FGP could be because the respondents answered these items from the use of non-FGP for all radiographies (not only the relatively simple small bone examinations). The items of the concept behavioural intention are formulated in that way (see Appendix A).

The results of SI indicate a rather neutral opinion. In several institutions, monitoring or support by the head radiographer and radiologists is rare (Germonpré et al., 2016). Therefore, it is possible that the staff in those institutions do not feel social pressure to use non-FGP. A second probable explanation is the fact that the use of non-FGP is not obligatory in most hospitals (Germonpré et al., 2016), as the impact of SI is found to be bigger in the context of nonvoluntary use (Venkatesh et al., 2003).

As predicted, PE, EE and SI all significantly affect the behavioural intention to use non-FGP. The fourth hypothesis that behavioural intention had a significant effect on the actual use was confirmed as well. 
In contrast with earlier studies (Venkatesh et al., 2003; Kijsanayotin et al., 2009; Liu et al., 2014) EE was found to be the strongest predictor of behavioural intention, although closely followed by PE. The fact that both constructs are found to be important predictors is consistent with prior findings (Venkatesh et al., 2003; Chang et al., 2007). It is important to keep in mind that the items representing PE were formulated in favour of FGP. Results could have been different if the items would have estimated participants' level of agreement on statements about the usefulness of non-FGP.

The third predictor, SI, also seemed to have a direct effect on behavioural intention, but the effect was only marginally significant. This is consistent with previous findings (Chang et al., 2007; Kijsanayotin et al., 2009).

As a last step, a moderation analysis was performed. Only the hypothesis that individualised consideration moderates the effect of SI on behavioural intention was confirmed by the results.

As expected the effect of social influence was strengthened or weakened dependent on how high one's direct supervisor scores on individualised consideration according to the individual's perception. The results indicated that a subject, who perceives his or her direct supervisor as giving frequently individual attention and support, will be more influenced by the opinion of important others. Given that leadership involves power and social influence (Gabel, 2012), a possible explanation is evident. Subjects who perceive their supervisor as more involved, will possibly experience more social pressure.

We were unable to demonstrate that leadership moderates the effects of PE and EE on behavioural intention. These results are contradictory to the hypotheses. Leaders, who make time for coaching moments and pay attention to individual needs, were expected to teach and reinforce their followers (Avolio and Bass, 1995). This study shows that the participants' perception of their supervisor did not change the effect of PE and EE. In order to find explanations for these results, more information about the leaders' work and his or her interaction with the team concerning the use of non-FGP for radiographs should be collected.

\subsection{Strengths, limitations and practical implications}

The first strength of this research is the selection of the sample. A randomly chosen sample is representative for the population. The response rate is the second strength of the study. Almost $60 \%$ of the distributed questionnaires were returned.

The first limitation of this study was the formulation of some items in the questionnaire. Problems arose to interpret the effect of PE. For a better understanding or a possible other result, the items representing PE should be formulated in favour of non-FGP. A second limitation was the impossibility to draw causal conclusions due to the cross-sectional design of this study. In order to study the causal relationship between behavioral intention and actual use better, a longitudinal research design would be more suitable. Social desirability can be seen as a third potential limitation of this paper. To avoid people from choosing the most desirable option on a statement, other methods should be used to measure behaviour.
This study has several practical implications. Since previous studies found that training of the staff is critical in adopting a new system (Calman et al., 2007), and more specifically determines if fluoroscopy is used to position the patient (Dierckx et al., 2005), results of this study are remarkable. Approximately two thirds of the participants did not attend an external training. Based on previously mentioned studies and this finding, hospitals should possibly invest in extra programmes for its staff. A second implication of this study is a managerial tool at the same time. Since PE, EE as well as SI are found to be significant predictors for the behavioural intention to use anatomic orientation points to position the patient, these insights can be used by the radiologists or by the head of the radiology department.

\section{Conclusion}

The results of this study indicate a relatively low use of non-FGP in Belgian radiology departments. Although staff training is critical to position the patient without fluoroscopy, results indicate a low percentage of participants who completed an external programme. This is an important practical implication of this study. Subsequently, the first research question is examined. Effort expectancy is found to be the strongest predictor of the behavioural intention to use nonFGP, closely followed by performance expectancy. The third predictor, social influence, also significantly affects the intention to use non-FGP. Those findings can be a helpful tool for radiologists and head radiographers to stimulate the staff to position patients for radiographic examinations only by using their skills and anatomic orientation points. When examining the effect of leadership, results show a marginally significant moderation effect of individualised consideration on the effect of social influence on behavioural intention. The findings of this research are considered to provide important guidelines for making future decisions in the Belgian radiology departments.

\section{References}

American Society of Radiologic Technologists. 2017. ASRT Position Statements. Available from https://www.asrt.org/docs/defaultsource/governance/hodpositionstatements.pdf.

Avolio BJ, Bass BM. 1995. Individual consideration viewed at multiple levels of analysis: A multi-level framework for examining the diffusion of transformational leadership. Leadersh. Q. 6(2): 199-218.

Avolio BJ, Bass BM. 2004. Multifactor Leadership Questionnaire: Manual and Sampler Set. Redwood City, CA: Mind Garden.

Berrington de González A, Darby S. 2004. Risk of cancer from diagnostic X-rays: Estimates for the UK and 14 other countries. Lancet 363(9406): 345-351.

Bontrager KL, Lampignano JP. 2014. Bontrager's Handbook of Radiographic Positioning and Techniques. St. Louis: Mosby Elsevier.

Calman N, Kitson K, Hauser D. 2007. Using information technology to improve health quality and safety in community health centers. Progr. Community Health Partnersh. 1(1): 83-88.

Chang I, Hwang H, Hung W, Li Y. 2007. Physicians' acceptance of pharmacokinetics-based clinical decision support systems. Expert Syst. Appl. 33(2): 296-303. 
Chau PYK, Hu PJ. 2001. Information technology acceptance by individual professionals: A model comparison approach. Decis. Sci. 32(4): 699-719.

Ciraj-Bjelac O, et al. 2011. Radiation protection of patients in diagnostic radiology: Status of practice in five Eastern-Europe countries, based on IAEA project. Eur. J. Radiol. 79: 70-73.

Deprez T, Schrijver L, Pauwels H, Bosmans H, Marchal G. 2001. Can patient positioning using an ultrashort fluoroscopic pulse be justified? In: IAEA, Radiological Protection of Patients in Diagnostic and Interventional Radiology, Nuclear Medicine \& Radiotherapy, Vienna, Austria, March 26-30, 2001, pp. 89-92.

Dierckx D, Constales K, Gerardy N, Goegebuer T, Persyn K. 2005. Patient dosimetry measurement in 50 radiology departments in Belgium. Radiat. Prot. Dosim. 117: 135-138.

Gabel S. 2012. Power, leadership and transformation: The doctor's potential for influence. Med. Educ. 46: 1152-1160.

Germonpré S, Gemmel P, Beeckman D, Trybou J. 2016. Determinants of using (non-)fluoroscopy-guided positioning for radiographs: A case study research. J. Radiol. Prot. 36(3): 667-679.

Haynes K, Curtis T. 2009. Fluoroscopic vs. Blind positioning: Comparing entrance skin exposure. Radiol. Technol. 81(1): 15-23.

International Atomic Energy Agency. 2015. Radiation protection in medicine. Setting the scene of the next decade. In: Proceedings of an International Conference, Bonn, 3-7 December 2012, International Atomic Energy Agency, Vienna. Available from http://wwwpub.iaea.org/MTCD/publications/PDF/Pub1663_web.pdf.

Jiang HJ, Lockee C, Bass K, Fraser I. 2008. Board engagement in quality: Findings of a survey of hospital and system leaders. $J$. Healthc. Manag. 53(2): 121-134.

Kijsanayotin B, Pannarunothai S, Speedie SM. 2009. Factors influencing health information technology adoption in Thailand's community health centers: Applying the UTAUT model. Int. J. Med. Inform. 78(6): 404-416.

Kroch E, et al. 2006. Hospital boards and quality dashboards. $J$. Patient Saf. 2(1): 10-19.

Liu L, Cruz AM, Rincon AR, Buttar V, Ranson Q, Goertzen D. 2014. What factor determine therapists' acceptance of new technologies for rehabilitation-A study using the Unified Theory of Acceptance and Use of Technology (UTAUT). Disabil. Rehabil. 37(5): 447-455.

Nunnally JC. 1978. Psychometric Theory. New York: McGraw-Hill.

Pynoo B, Devolder P, Duyck W, van Braak J, Sijnave B, Duyck P. 2012. Do hospital physicians' attitude change during PACS implementation? A cross-sectional acceptance study. Int. J. Med. Inform. 81: 88-97.

Saunders M, Budden A, MacIver F, Teunis M, Warrenforward H. 2005. Dose implications of fluoroscopy-guided positioning (FGP) for lumbar spine examinations prior to acquiring plain film radiographs. Br. J. Radiol. 78: 130-134.

Sheppard BH, Harwick J, Warshaw PR. 1988. The theory of reasoned action: A meta-analysis of past research with recommendation for modifications and future research. J. Consum. Res. 15: 325-343.
Statkiewicz-Sherer MA, Visconti PJ, Ritenour ER, Haynes KW. 2014. Radiation Protection in Medical Radiography. Maryland Heights: Mosby, Elsevier.

Venkatesh V, Morris MG, Davis GB, Davis FD. 2003. User acceptance of information technology: Toward a unified view. MIS Q. 27(3): 425-478.

Vrijens F, et al. 2012. Performance of the belgian health system. KCE Report 196C, 2012. Available from https://kce.fgov.be/sites/ default/files/page documents/KCE 196C Health system per formance_0.pdf.

\section{Appendix A}

Performance expectancy

"I think fluoroscopy is useful in my job"

"Using fluoroscopy enables me to work more quickly"

"Using fluoroscopy increases my productivity"

Effort expectancy

"The techniques of non-FGP are easy to understand"

"I master the techniques of non-FGP well"

"I think non-FGP is simple"

"I think acquiring the techniques of non-FGP is simple"

Social influence

"My colleagues think I should always use non-FGP"

"The radiologists think I should always use non-FGP" FGP"

"My direct supervisor supports me in the use of nonFGP"

"In general, the department supports the use of non-

Behavioural intention

"I have the intention to use non-FGP for all radiographs in the next months"

"I am planning to use non-FGP for all radiographs in the next months"

"I predict I will use non-FGP for all radiographs in the next months"

Use months?"

"To what extent did you use non-FGP during the last few

Individualised consideration

"My direct supervisor pays attention to each one individually"

"My direct supervisor confirms my strengths"

"My direct supervisor helps me to develop my competences"

"My direct supervisor makes a distinction between his employees"

Cite this article as: Germonpré S, Vermaut G, Trybou J. 2019. The acceptance of radiographers to use Non-fluoroscopy Guided Positioning and the influence of leadership: a survey research. Radioprotection, https://doi.org/10.1051/radiopro/2019028. 